\title{
Unobtrusive Analysis of Group Interactions without Cameras
}

\author{
Indrani Bhattacharya \\ Rensselaer Polytechnic Institute \\ Troy, New York \\ bhatti@rpi.edu
}

\begin{abstract}
Group meetings are often inefficient, unorganized and poorly documented. Factors including "group-think", fear of speaking, unfocused discussion, and bias can affect the performance of a group meeting. In order to actively or passively facilitate group meetings, automatically analyzing group interaction patterns is critical. Existing research on group dynamics analysis still heavily depends on video cameras in the lines of sight of participants or wearable sensors, both of which could affect the natural behavior of participants. In this thesis, we present a smart meeting room that combines microphones and unobtrusive ceiling-mounted Time-of-Flight (ToF) sensors to understand group dynamics in team meetings. Since the ToF sensors are ceiling-mounted and out of the lines of sight of the participants, we posit that their presence would not disrupt the natural interaction patterns of individuals. We collect a new multi-modal dataset of group interactions where participants have to complete a task by reaching a group consensus, and then fill out a post-task questionnaire. We use this dataset for the development of our algorithms and analysis of group meetings. In this paper, we combine the ceiling-mounted ToF sensors and lapel microphones to: (1) estimate the seated body orientation of participants, (2) estimate the head pose and visual focus of attention (VFOA) of meeting participants, (3) estimate the arm pose and body posture of participants, and (4) analyze the multimodal data for passive understanding of group meetings, with a focus on perceived leadership and contribution.
\end{abstract}

\section{CCS CONCEPTS}

- Human-centered computing $\rightarrow$ Collaborative and social computing systems and tools; • Applied computing $\rightarrow P s y$ chology;

\section{KEYWORDS}

Multimodal sensing, smart rooms, time-of-flight sensing, multiple person tracking, head pose estimation, arm and body posture, group meeting analysis

\section{ACM Reference Format:}

Indrani Bhattacharya. 2018. Unobtrusive Analysis of Group Interactions without Cameras. In 2018 International Conference on Multimodal Interaction

Permission to make digital or hard copies of all or part of this work for personal or classroom use is granted without fee provided that copies are not made or distributed for profit or commercial advantage and that copies bear this notice and the full citation on the first page. Copyrights for components of this work owned by others than the author(s) must be honored. Abstracting with credit is permitted. To copy otherwise, or republish, to post on servers or to redistribute to lists, requires prior specific permission and/or a fee. Request permissions from permissions@acm.org.

ICMI '18, October 16-20, 2018, Boulder, CO, USA

(C) 2018 Copyright held by the owner/author(s). Publication rights licensed to ACM. ACM ISBN 978-1-4503-5692-3/18/10 ..\$15.00

https://doi.org/10.1145/3242969.3264973
(ICMI '18), October 16-20, 2018, Boulder, CO, USA. ACM, New York, NY, USA, 5 pages. https://doi.org/10.1145/3242969.3264973

\section{INTRODUCTION}

Studying group dynamics is an inherently difficult problem, because events in a group process can change at a millisecond time frame, making them extremely difficult to measure and quantify $[12,26,28]$. The cause of a particular behavior could be a culmination of several factors including previous behaviors, individual and group characteristics, context, and group composition [11, 17]. Thus, researchers studying group dynamics need to account for the temporal and spatial resolution of events occurring in a group at a very fine granularity; this quickly results in social psychologists struggling with huge volumes of data.

Non-verbal behavior contains rich information about the nature of interaction. However, manually coding such nonverbal behavior like location, gaze, gestures, head and body movements, posture, speaker segmentation, interruptions and so on, is cumbersome, time-consuming and subjective. Additionally, manual coders may not be able to capture the full range of nonverbal behavior due to the positioning of witness cameras and the typical temporal subsampling of frames. Efforts to make manual coding easier (e.g., asking participants to wear brightly colored vests [30]) may also make a discussion less natural.

Today, the availability of new sensors and advanced computer vision and signal processing algorithms allow for the automatic analysis of non-verbal behavior that is important for the analysis of social interaction. Team science research is now catching up to the promise of these technologies, using a mix of off-the-shelf cameras, microphones, and Microsoft Kinects. For example, Oertel et al. [34] developed a multi-modal corpus to analyze conversational behavior in group interviews. The setup required 4 participants to be seated symmetrically around a round table and each participant was recorded by a dedicated sensor array that included: (1) a close-talking mono-directional microphone to record high quality speech, (2) a Windows Kinect, placed $0.8 \mathrm{~m}$ in front of the participant on the table to capture RGBD information, and (3) a GoPro camera, tied to the Kinect, to record high quality video data to complement the low quality RGB data from the Kinect for accurate gaze estimation. While the system provides valuable information, the array of cords, memory cards, and external PCs is not really compact and the position of the sensors in the lines of sights of participants may appear obtrusive to the participants. Similarly, recent work by Müller et al. for understanding rapport in conversations [32] is based on a multi-modal dataset in which each participant is recorded by two external cameras and an omnidirectional microphone. Other recent systems for collecting team research data include those used in the ELEA corpus [42] (7 cameras, 2 laptops, and a microphone array), the ICSI meeting corpus [21] (head-worn 
and table-top microphones), the ISL meeting corpus [9] (microphones), the AMI corpus [24] (video cameras and microphones), the ATR database [10] (small 360-degree camera surrounded by an array of high-quality directional microphones), and the NTT corpus [37-39] (video cameras, microphones and wearable sensors). The Human Dynamics Group at the MIT Media lab has used wearable devices called "sociometric badges" [25] for studying group interaction. These badges collect the wearers' coarse physical locations, physical proximity to other people, physical activity, and conversational time (but not actual speech).

All these systems use front facing cameras/ Kinects or wearable sensors. However, the presence of visible cameras can alter participants' natural behavior [46], and having unusual sensors directly in front of ones' face or in the line of sight may also inhibit natural individual behavior or group interactions. We posit that a room in which the participants are as unaware of being sensed as possible is best for studying natural group dynamics.

Ideally, we want a smart environment that can unobtrusively understand the location, body orientation, head pose, arm pose, body posture, and non-verbal/ verbal speech of all participants in a group meeting without making them feel uncomfortable or inhibited. In this paper, we propose a multimodal-sensor-enabled Smart Conference Room (SCR) to study human interaction patterns in group meetings using ceiling mounted Time-of-Flight (ToF) sensors and lapel microphones [1]. This $11^{\prime} \times 28^{\prime}$ conference room, located at Rensselaer Polytechnic Institute, has 18 ceiling-mounted IRMA Matrix low-resolution ToF sensors and 2 ceiling-mounted high-resolution Kinects positioned over the edge of the table. The depth maps formed by the ToF sensors, combined with non-verbal audio information, can be processed by computer vision, signal processing and machine learning algorithms to understand group interaction patterns at a fine spatial and temporal resolution. We use ToF sensors because (1) the distance maps returned allow for the creation of 3D point clouds of the environment, and (2) they are robust to ambient lighting changes and color/reflectivity of participant's clothing.

The lower resolution sensors can be used to accurately track occupants in the room and understand their coarse body pose and orientation. However, they cannot be used to understand the head pose of occupants; hence we use the higher resolution ToF sensors to understand the head pose, visual focus of attention (VFOA), arm pose and body posture of meeting participants.

\section{RESEARCH PLAN AND CONTRIBUTIONS}

\subsection{Experimental design and building the dataset}

There is no existing dataset to study group interactions using ceiling-mounted depth sensors to the best of our knowledge. In order to build a structured, well-defined dataset to study group interactions, our initial task was to build a corpus of multi-modal group meeting data in the Smart Conference Room. We recorded 22 Lunar Survival Task experiments [15] in the SCR with 86 participants, which form our current dataset for passive meeting understanding. The Lunar Survival Task is a widely-used group discussion task that assesses the effects of deliberation processes on decision-making quality. In small groups of 3-5, participants discuss a hypothetical survival scenario and rank the value of supplies that may aid in their survival. Each discussion lasts from 10-15 minutes, after which the participants are asked to complete a post-task questionnaire. The post-task questionnaire asks the participants about their age, gender, who they thought was the emergent leader, who they thought contributed the most, how well they knew each other, and whether the discussion was engaging/boring, comfortable/awkward. The recorded multi-modal data included the lower resolution depth map from the 18 overhead IRMA Matrix ToF sensors, the higher resolution depth map from the 2 overhead Kinect sensors, and audio information collected from individual lapel microphones on each participant. We also collected reference video using two video cameras at both ends of the room. The video camera data is not used for any algorithm development and is purely used for ground truth determination.

\subsection{Estimation of body orientation of seated individuals using low resolution overhead ToF sensors}

The low resolution depth sensors are used to track people in real time by performing background subtraction and then comparing each person blob with the person blobs in the previous frame to find the closest match using the Hungarian algorithm. Temporary false alarms or misses are handled using logic that people cannot spontaneously appear or disappear from the middle of the room. As shown in our group's previous work [7,23], this relatively simple approach is quite accurate for our purposes; more complex and computationally demanding Kalman or particle filters for tracking are not necessary. A maximum likelihood estimator based on features extracted from the time series of ToF measurements is used to classify human pose into sitting, standing or walking states.

Our first contribution is the estimation of the relative orientations of people during a group interaction, an important non-verbal cue. Given a meeting scenario, we want to estimate the body orientation of all seated individuals at each ToF frame. We consider 8 orientation classes, separated by $45^{\circ}$. The top-down views of the eight orientations and corresponding representative low resolution ToF images for an individual are shown in Figure 1.

Our body orientation estimation algorithm uses a Bayes classifier applied to measurements from the ToF sensors and the lapel microphones. The likelihood term is computed by applying compressed sensing techniques to the ToF depth map. The prior term is calculated dynamically at each frame after extracting the speaker identity from the audio, combined with knowledge of the relative positions of the participants.

Figure 2 illustrates a sample result of the orientation classification algorithm. Figure 2a is the reference view, Figure $2 b$ is the corresponding raw ToF data, and Figure 2c shows the location, speaker, and estimated body orientations. The algorithm works with $80.8 \%$ accuracy to exactly classify seated orientations and with $98 \%$ accuracy to classify orientations with an error of 1 orientation class $\left( \pm 45^{\circ}\right)$. A short video clip with the reference camera view, the ToF raw output, and the algorithm results are available at the link https://youtu.be/Hm98ZEqjAtk. The work was published as [5]. 


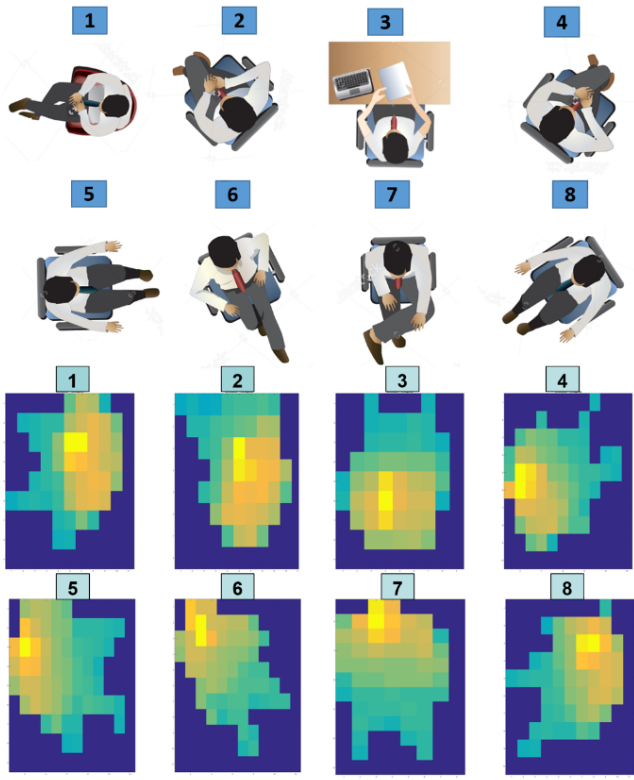

Figure 1: Top-down views of orientation classes and corresponding actual ToF images.

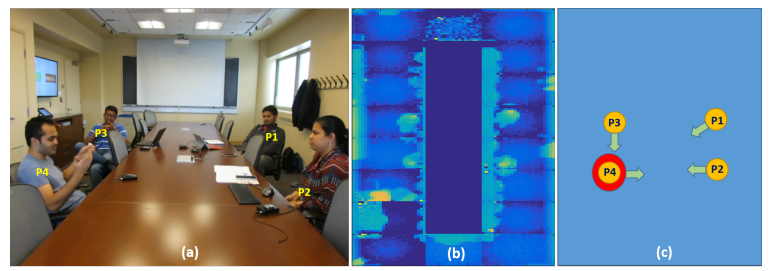

Figure 2: (a) Camera view, (b) Raw data from the ToF sensors stitched to form a depth map of the room, (c) Results of the orientation estimation algorithm. The red circle indicates the speaker, detected from the microphone recordings. The yellow arrows indicate the automatically estimated body orientations.

\subsection{Estimation of head pose and visual focus of attention (VFOA) from higher resolution overhead ToF sensors}

The gaze and visual focus of attention (VFOA) of people are important nonverbal cues that convey rich information about the nature of the conversation. For example, if two people share mutual gaze during a verbal communication, it could indicate they are both engaged in the conversation. The problem of estimating VFOA of participants from non-verbal cues is an active area of research $[2,13,36,37,45]$.

As opposed to existing works, we use a fusion of overhead depth sensors and synchronized speaker identification information for understanding VFOA in the context of a group meeting. For computation of the head pose, we compute 3D point clouds from the depth map of each Kinect, and apply a rigid transformation that optimally aligns the two point clouds in the least squares sense to build a combined 3D point cloud of the entire scene. The points corresponding to the head of each participant are determined based on a dynamic thresholding criterion, and an ellipsoid is fit to the 3D head points of each seated individual. The head pose is estimated for each participant by tracking the axes of the ellipsoids, together with appropriate filtering. Our VFOA estimator is a Bayes classifier that uses both the depth information from the Kinect sensors and the synchronized speaker identification information from the microphone recordings. The likelihood term is computed from the head pose measurements while the prior term is computed dynamically at each frame based on the speaker information. Figure 3 shows a frame with the estimated head pose and VFOA superimposed on the $3 \mathrm{D}$ point cloud, together with the active speaker, if any. A short video clip illustrating the VFOA estimation on a meeting segment is at https://youtu.be/ki5UJPSOZdE. This work was published as [6].

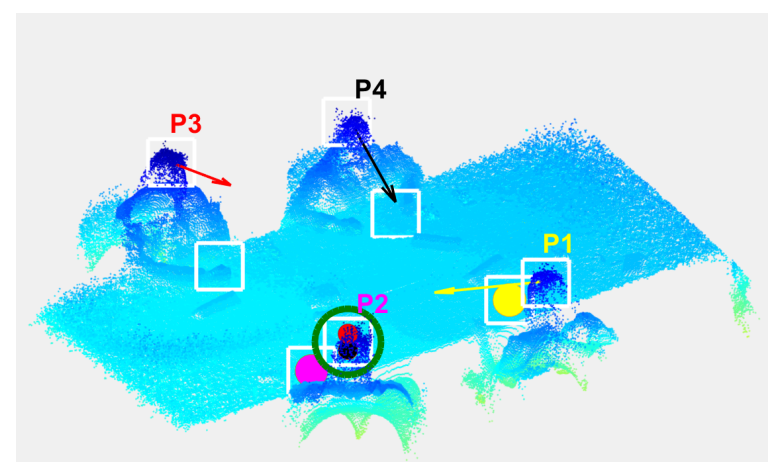

Figure 3: The estimated VFOA of the participants. The white boxes represent the possible targets (either of the 4 participants or the paper in front of them); the colored arrows and the dots represent the estimated VFOAs of each participant. The green circle represents the active speaker, if any.

The Bayes classifier combining the head pose and synchronized speaker identification gives a VFOA classification accuracy of $48.35 \%$. In comparison, Jayagopi et al. reported an accuracy of $42 \%$ in similar group meeting settings with the same VFOA target locations using front-facing video cameras [22].

Since the orientation of the shoulders is an important indicator of the body orientation, we hypothesize that including both the shoulder and head regions in a supervised learning framework would help improve the VFOA estimation accuracy. In future thesis work, we plan to use a convolutional neural network (CNN) based framework on the head and shoulder 3D points to improve the VFOA estimation accuracy.

\subsection{Estimation of arm pose and body posture in the context of group meetings}

Low-level coordination, which includes coordination using nonverbal cues, has been shown to facilitate smooth and focused interaction, and this in turn provides a cooperative foundation for faceto-face conversation. Several psychological variables like "trust", "liking" and "rapport" can be considered as proxies for cooperation. 
In order to study the non-verbal correlates of rapport, Harrigan et al. [16] manually coded non-verbal behavior including proximity, body posture, gaze orientation, hand movements, and head and face movements. They considered trunk angle (\% of time in forward, upright and backward positions, overall means and frequencies of shifts) and arm position (\% of time in open and crossed (hands together), symmetrical and asymmetrical arm positions) as important indicators of positivity, coordination and openness. Lee et al. [27] found a set of four non-verbal cues - face touching, arms crossed, leaning backwards, and hand touching - to be representative of lower levels of trust.

A current research direction of my thesis is the development of an algorithm to automatically detect arm pose (closed arms, arms on table, arms on body, arms in conversational gesture) and body trunk pose (leaning, upright, and backwards) from overhead depth measurements taken from the two Kinect sensors. Figure 4 shows a mock-up view of the kind of classification of arm and trunk posture we want to estimate from the Kinect depth data.

While there is substantial literature in the field of computer vision to track hands [35] and arm and body posture [14, 20, 33, $40,41]$, we must develop/modify existing algorithms that can work with our setup and overhead depth information.

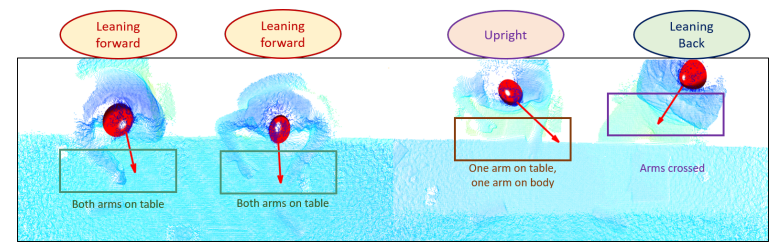

Figure 4: Top-down view of the 3D point cloud of the meeting participants, with fitted ellipsoids and head pose directions. Our proposed extension is a system that would automatically understand the arm pose and the body trunk pose.

\subsection{Analysis of multi-modal data for passive understanding of group meetings, with focus on perceived emergent leadership and contribution}

Existing research using multiple cameras and front-facing Kinects and/or wearable sensors addresses fine-grained analyses of group interaction patterns and the automatic determination of social constructs such as agreement/disagreement [8], cohesion [18], dominance [19, 22], leadership [3, 4, 44] or emotion [31] in group interactions. In [29], the authors discuss some of these automatic analysis techniques and how automating the process saves hours of manual annotation effort. Our next research question is whether the ceiling-mounted depth sensors can provide equally good analysis of group interactions, without being obtrusive/inhibiting. We focus on two social psychology variables: perceived emergent leadership and perceived contribution. The post-task questionnaire ratings serve as our target variables. Since each group member rates the leadership and contribution of all other group members on a 5-point scale, we compute an individual's perceived leadership score as the average of all leadership scores they receive from their group.
We compute several non-verbal metrics based on extracted VFOA (attention received, attention given, etc.) and speaker segmentation (interruption patterns, speaking lengths, etc.). We also automatically generate a transcript from the audio files for each speaker using IBM Watson's Speech-to-Text API [43], and extract verbal metrics related to the mentions of items and item/ranking pairs.

We then studied the correlation between the metrics and the target variables and used multiple linear regression to investigate the capability of the extracted metrics to predict leadership and contribution. From our initial experiments on 10 meetings, we found that a combination of all the visual, non-verbal and verbal metrics can explain $65 \%(F=1.39, p=0.26)$ of the variability of the leadership scores. Similarly, all the metrics combined can explain $63 \%(F=1.27$, $p=0.32$ ) of the variability of the contribution scores. We found that by combining the visual, non-verbal and verbal metrics, we were able to predict the perceived emergent leader with an accuracy of $90 \%$ and the major contributor with an accuracy of $100 \%$. This result is promising and shows that even without front-facing video cameras, we can analyze group meetings in terms of leadership and contribution to a plausible level of accuracy. This work was published as [6].

As a proposed extension to this analysis of perceived leadership and contribution, we want to include additional audio-visual metrics and metrics based on head and body movements and arm and body posture. While it is difficult to understand subtle head and body movements from overhead depth information, we posit that significant events of head and body movement can be detected from the overhead Kinect depth information. Significant levels of body movement can be an indication of agitation or restlessness. Head movements can be due to nodding/shaking to indicate approval/disapproval.

\subsection{Progress to date and expected contributions}

The expected completion date of the thesis is June 2019. The existing and future contributions of the thesis include:

- Occupancy tracking using low-resolution ceiling-mounted ToF sensors (published as [7])

- Estimation of seated body orientation (published as [5])

- Estimation of head pose and VFOA (Bayesian method - published as [6]; CNN-based method - under development, expected completion by October 2018)

- Estimation of arm pose and body posture (under development, expected completion by April 2019)

- Analysis of multi-modal data (initial analysis - published as [6]; proposed extension expected completion by June 2019)

\section{ACKNOWLEDGMENTS}

This work was supported by the NSF under award IIP-1631674 from the PFI:BIC program, by the NSF under cooperative agreement EEC0812056, and by New York State under NYSTAR contract C090145. I want to extend my heartfelt thanks to my adviser Dr. Richard J. Radke for his supervision, motivation and constant support. I also extend my thanks to Michael Foley, Dr. Brooke Foucault Welles and Dr. Christoph Riedl from Northeastern University and Ni Zhang, Tongtao Zhang, Devavrat Jivani, Gyanendra Sharma and Dr. Heng Ji from Rensselaer Polytechnic Institute. 


\section{REFERENCES}

[1] S Afshari, TK Woodstock, MHT Imam, S Mishra, AC Sanderson, and RJ Radke. 2015. The Smart Conference Room: An Integrated System Testbed for Efficient, Occupancy-aware Lighting Control. In ACM Int. Conf. Embedded Syst. EnergyEfficient Built Environments.

[2] Sileye O Ba and Jean-Marc Odobez. 2011. Multiperson visual focus of attention from head pose and meeting contextual cues. IEEE Trans. Pattern Anal. and Mach. Intell. 33, 1 (2011), 101-116.

[3] Cigdem Beyan, Francesca Capozzi, Cristina Becchio, and Vittorio Murino. 2017 Multi-task learning of social psychology assessments and nonverbal features for automatic leadership identification. In Proc. 19th Int. Conf. Multimodal Interaction. ACM.

[4] Cigdem Beyan, Francesca Capozzi, Cristina Becchio, and Vittorio Murino. 2018 Prediction of the leadership style of an emergent leader using audio and visual nonverbal features. IEEE Trans. Multimedia 20, 2 (2018), 441-456.

[5] Indrani Bhattacharya, Noam Eshed, and Richard J Radke. 2017. PrivacyPreserving Understanding of Human Body Orientation for Smart Meetings. In Int. Conf. Comput. Vision Pattern Regognition Workshops. IEEE.

[6] Indrani Bhattacharya, Michael Foley, Ni Zhang, Tongtao Zhang, Christine Ku, Cameron Mine, Heng Ji, Christoph Riedl, Brooke F. Welles, and Richard J. Radke. 2018. A multimodal-sensor-enabled room for unobtrusive group meeting analysis. In Proc. Int. Conf. Multimodal Interaction. ACM.

[7] Indrani Bhattacharya and Richard J Radke. 2016. Arrays of single pixel time-offlight sensors for privacy preserving tracking and coarse pose estimation. In Proc. Winter Conf. Appl. Comput. Vision. IEEE.

[8] Konstantinos Bousmalis, Marc Mehu, and Maja Pantic. 2013. Towards the automatic detection of spontaneous agreement and disagreement based on nonverbal behaviour: A survey of related cues, databases, and tools. Image and Vision Computing 31, 2 (2013), 203-221.

[9] Susanne Burger, Victoria MacLaren, and Hua Yu. 2002. The ISL meeting corpus The impact of meeting type on speech style. In INTERSPEECH. Denver, CO.

[10] Nick Campbell, Toshiyuki Sadanobu, Masataka Imura, Naoto Iwahashi, Suzuki Noriko, and Damien Douxchamps. 2006. A multimedia database of meetings and informal interactions for tracking participant involvement and discourse flow. In Proc. Int. Conf. Lang. Resources Evaluation. Genoa, Italy.

[11] Ming Ming Chiu and Nale Lehmann-Willenbrock. 2016. Statistical discourse analysis: Modeling sequences of individual actions during group interactions across time. Group Dynamics: Theory, Research, and Practice 20, 3 (2016), 242.

[12] Matthew A Cronin, Laurie R Weingart, and Gergana Todorova. 2011. Dynamics in groups: Are we there yet? Academy of Management Annals 5, 1 (2011), 571-612.

[13] Daniel Gatica-Perez, Alessandro Vinciarelli, and Jean-Marc Odobez. 2014. Nonverbal behavior analysis. In Multimodal Interactive Syst. Manage. EPFL Press, 165-187.

[14] Liuhao Ge, Hui Liang, Junsong Yuan, and Daniel Thalmann. 2018. Real-time 3D hand pose estimation with 3D Convolutional Neural Networks. IEEE Trans. Pattern Anal. Mach. Intell. (2018).

[15] Jay Hall and Wilfred Harvey Watson. 1970. The effects of a normative intervention on group decision-making performance. Human Relations 23, 4 (1970), 299-317.

[16] Jinni A Harrigan, Thomas E Oxman, and Robert Rosenthal. 1985. Rapport expressed through nonverbal behavior. 7. Nonverbal Behavior 9, 2 (1985), 95-110.

[17] Benjamin Herndon and Kyle Lewis. 2015. Applying sequence methods to the study of team temporal dynamics. Organizational Psychology Review 5, 4 (2015) 318-332.

[18] Hayley Hung and Daniel Gatica-Perez. 2010. Estimating cohesion in small groups using audio-visual nonverbal behavior. IEEE Trans. Multimedia 12, 6 (2010), $563-$ 575 .

[19] Hayley Hung, Yan Huang, Gerald Friedland, and Daniel Gatica-Perez. 2011. Estimating dominance in multi-party meetings using speaker diarization. IEEE Trans. Audio, Speech, and Language Process. 19, 4 (2011), 847-860.

[20] Umar Iqbal, Pavlo Molchanov, Thomas Breuel, Juergen Gall, and Jan Kautz. 2018. Hand pose estimation via Latent 2.5D heatmap regression. arXiv preprint arXiv:1804.09534 (2018)

[21] Adam Janin, Don Baron, Jane Edwards, Dan Ellis, David Gelbart, Nelson Morgan, Barbara Peskin, Thilo Pfau, Elizabeth Shriberg, Andreas Stolcke, et al. 2003. The ICSI meeting corpus. In Int. Conf. Acoust., Speech, and Signal Process. Hong Kong, China.

[22] Dineshbabu Jayagopi, Dairazalia Sanchez-Cortes, Kazuhiro Otsuka, Junji Yamato, and Daniel Gatica-Perez. 2012. Linking speaking and looking behavior patterns with group composition, perception, and performance. In Proc. 14th ACM Int. Conf. Multimodal Interaction. ACM.

[23] Li Jia and Richard J Radke. 2014. Using Time-of-Flight measurements for privacypreserving tracking in a smart room. IEEE Trans. Ind. Informat. 10, 1 (2014), 689-696.

[24] Natasa Jovanovic, Rieks op den Akker, and Anton Nijholt. 2006. A corpus for studying addressing behaviour in multi-party dialogues. Language Resources and Evaluation 40, 1 (2006), 5-23.
[25] Taemie Kim, Erin McFee, Daniel Olguin Olguin, Ben Waber, Alex Pentland, et al. 2012. Sociometric badges: Using sensor technology to capture new forms of collaboration. 7. Organizational Behavior 33, 3 (Jan. 2012), 412-427.

[26] Steve WJ Kozlowski. 2015. Advancing research on team process dynamics: Theoretical, methodological, and measurement considerations. Organizational Psychology Review 5, 4 (2015), 270-299.

[27] Jin Joo Lee, Brad Knox, Jolie Baumann, Cynthia Breazeal, and David DeSteno. 2013. Computationally modeling interpersonal trust. Frontiers in Psychology 4 (2013), 893

[28] Roger Th AJ Leenders, Noshir S Contractor, and Leslie A DeChurch. 2016. Once upon a time: Understanding team processes as relational event networks. Organizational Psychology Review 6, 1 (2016), 92-115.

[29] Nale Lehmann-Willenbrock, Hayley Hung, and Joann Keyton. 2017. New frontiers in analyzing dynamic group interactions: Bridging social and computer science. Small Group Research 48, 5 (2017), 519-531.

[30] Shobhit Mathur, Marshall Scott Poole, Feniosky Pena-Mora, Mark HasegawaJohnson, and Noshir Contractor. 2012. Detecting interaction links in a collaborating group using manually annotated data. Social Networks 34, 4 (2012), 515-526.

[31] Wenxuan Mou, Hatice Gunes, and Ioannis Patras. 2016. Alone versus in-a-group: A comparative analysis of facial affect recognition. In Proc. ACM Multimedia Conf. ACM, 521-525.

[32] Philipp Müller, Michael Xuelin Huang, and Andreas Bulling. 2018. Detecting low rapport during natural interactions in small groups from non-Verbal behaviour. arXiv preprint arXiv:1801.06055 (2018).

[33] Markus Oberweger and Vincent Lepetit. 2017. DeepPrior++: Improving fast and accurate 3D hand pose estimation. In Int. Conf. Comput. Vision Workshops, Vol. 840. 2.

[34] Catharine Oertel, Kenneth A Funes Mora, Samira Sheikhi, Jean-Marc Odobez, and Joakim Gustafson. 2014. Who will get the grant?: A multimodal corpus for the analysis of conversational behaviours in group interviews. In Proc. Workshop Understanding Modeling Multiparty, Multimodal Interactions. ACM.

[35] Iason Oikonomidis, Nikolaos Kyriazis, and Antonis A Argyros. 2011. Efficient model-based 3D tracking of hand articulations using Kinect.. In $B m V C$, Vol. 1. 3.

[36] Kazuhiro Otsuka, Shoko Araki, Kentaro Ishizuka, Masakiyo Fujimoto, Martin Heinrich, and Junji Yamato. 2008. A realtime multimodal system for analyzing group meetings by combining face pose tracking and speaker diarization. In Proc. Int. Conf. Multimodal Interfaces. ACM, Crete, Greece.

[37] Kazuhiro Otsuka, Hiroshi Sawada, and Junji Yamato. 2007. Automatic inference of cross-Modal nonverbal interactions in multiparty conversations: Who responds to whom, when, and how? From gaze, head gestures, and utterances. In Proc. Int. Conf. Multimodal Interfaces. ACM, Aichi, Japan.

[38] Kazuhiro Otsuka, Yoshinao Takemae, and Junji Yamato. 2005. A probabilistic inference of multiparty-conversation structure based on Markov-switching models of gaze patterns, head directions, and utterances. In Proc. 7th Int. Conf. Multimodal Interfaces. ACM.

[39] Kazuhiro Otsuka, Junji Yamato, Yoshinao Takemae, and Hiroshi Murase. 2006. Conversation scene analysis with dynamic Bayesian Network based on visual head tracking. In Proc. Int. Conf. Multimedia and Expo. IEEE, Toronto, ON, Canada.

[40] George Papandreou, Tyler Zhu, Liang-Chieh Chen, Spyros Gidaris, Jonathan Tompson, and Kevin Murphy. 2018. PersonLab: Person pose estimation and instance segmentation with a bottom-up, part-based, geometric embedding model. CoRR abs/1803.08225 (2018)

[41] George Papandreou, Tyler Zhu, Nori Kanazawa, Alexander Toshev, Jonathan Tompson, Chris Bregler, and Kevin P. Murphy. 2017. Towards accurate multiperson pose estimation in the wild. CoRR abs/1701.01779 (2017).

[42] Dairazalia Sanchez-Cortes, Oya Aran, and Daniel Gatica-Perez. 2011. An audio visual corpus for emergent leader analysis. In Workshop Multimodal Corpora Mach. Learning: Taking Stock and Road Mapping the Future. Alicante, Spain.

[43] George Saon, Gakuto Kurata, Tom Sercu, Kartik Audhkhasi, Samuel Thomas, Dimitrios Dimitriadis, Xiaodong Cui, Bhuvana Ramabhadran, Michael Picheny, Lynn-Li Lim, Bergul Roomi, and Phil Hall. 2017. English Conversational Telephone Speech Recognition by Humans and Machines. In Proc. INTERSPEECH.

[44] Stefan Scherer, Nadir Weibel, Louis-Philippe Morency, and Sharon Oviatt. 2012. Multimodal prediction of expertise and leadership in learning groups. In Int. Workshop Multimodal Learning Analytics.

[45] Rainer Stiefelhagen, Jie Yang, and Alex Waibel. 2002. Modeling focus of attention for meeting indexing based on multiple cues. Trans. Neural Netw. 13, 4 (2002), 928-938.

[46] Thomas J. L. van Rompay, Dorette J. Vonk, and Marieke L. Fransen. 2009. The eye of the camera: Effects of security cameras on prosocial behavior. Environment and Behavior 41, 1 (2009), 60-74. 